\title{
Detection of Anti-Leptospira IgM Antibody in Serum Samples of Suspected Patients Visiting National Public Health Laboratory, Teku, Kathmandu
}

\author{
Krishna Prasad Dahal, ${ }^{1}$ Supriya Sharma, ${ }^{1}$ Jeevan Bahadur Sherchand, ${ }^{2}$ \\ Bishnu Prasad Upadhyay, ${ }^{3}$ and Dwij Raj Bhatta ${ }^{1}$ \\ ${ }^{1}$ Central Department of Microbiology, Tribhuvan University, Kirtipur, Nepal \\ ${ }^{2}$ Tribhuvan University Teaching Hospital, Kathmandu, Nepal \\ ${ }^{3}$ National Public Health Laboratory, Teku, Nepal \\ Correspondence should be addressed to Krishna Prasad Dahal; cfckrismyth@gmail.com
}

Received 20 August 2016; Revised 5 November 2016; Accepted 13 November 2016

Academic Editor: Todd R. Callaway

Copyright (C) 2016 Krishna Prasad Dahal et al. This is an open access article distributed under the Creative Commons Attribution License, which permits unrestricted use, distribution, and reproduction in any medium, provided the original work is properly cited.

\begin{abstract}
Leptospirosis is a globally distributed zoonosis with varied clinical outcomes and multiorgan involvement in humans. In this study conducted from July 2011 to December 2011, 178 serum samples from patients suspected of leptospirosis were tested by Panbio IgM ELISA at National Public Health Laboratory, Kathmandu, out of which 51 (28.65\%) were positive for anti-Leptospira IgM antibody. Leptospirosis was more common in people in their 2nd and 3rd decades of their life which together comprised $56.86 \%$ of the total positive cases. Most of those tested positive were farmers followed by students and housewives. Both animal contact and water contact seemed to play significant roles in disease transmission. Symptoms were vague with the most common being fever, headache, myalgia, abdominal pain, vomiting, jaundice, and diarrhoea. Life style heavily dominated by agronomical and farming activities in Nepal is conducive to leptospirosis transmission. Leptospirosis seems to be a significant public health problem in Nepal but is underestimated. In resource poor countries like Nepal where laboratories performing MAT or maintaining cultures are rarely available, serological test like ELISA could well depict the scenario of the disease prevalence.
\end{abstract}

\section{Introduction}

Leptospirosis is a worldwide zooanthroponosis caused by pathogenic species of Leptospira [1]. Approximately half of the pathogenic serovars belong to L. interrogans or L. borgpetersenii [2]. The disease is of protean manifestation with outcomes ranging from an undifferentiated febrile illness to life threatening conditions such as Weil's disease and severe pulmonary haemorrhagic syndrome. Mortality from severe forms of the disease is about $5-40 \%$ and prompt diagnosis and early administration of antibiotics are critical in saving lives $[3,4]$. Pathogenic leptospires are wide spread in nature and are capable of surviving in both environment and renal tubules of the host harbouring them [5]. Many wild and domestic animals like rats, dogs, cattle, pigs, horses, and so forth are potential reservoirs of the causative spirochetes $[6,7]$. Infection occurs through contact with wild or domestic animals or exposure to soil or water contaminated by their urine [8].

Leptospirosis is difficult to diagnose because of its broad spectrum of symptoms and multiorgan involvement $[8,9]$. Culture needs prolonged time period of weeks while PCR requires special equipment, highly skilled personnel and has not been evaluated worldwide [10, 11]. Microscopic Agglutination Test (MAT) is restricted to laboratories that are capable of maintaining strains for preparations of live antigens $[8,12]$. ELISA uses a broadly reactive genus specific antigen to detect anti-Leptospira IgM and is a popular surrogate to MAT $[10,13]$.

Leptospirosis is highly prevalent in Asia Pacific Region and outbreaks in developing countries are most frequently related to normal daily activities, overcrowding, poor sanitation, and climactic condition [2]. The disease continues 
TABLE 1: Age wise distribution of leptospirosis.

\begin{tabular}{lccc}
\hline Age group & Number of cases & Number of positives (\%) & \% in total ELISA positives \\
\hline $0-10$ & 14 & $1(7.14)$ & 1.96 \\
$10-20$ & 36 & $9(25)$ & 35.29 \\
$20-30$ & 54 & $18(33.33)$ & 21.57 \\
$30-40$ & 29 & $11(37.93)$ & 13.73 \\
$40-50$ & 21 & $7(33.33)$ & 5.88 \\
$50-60$ & 11 & $3(27.27)$ & 3.92 \\
$60-70$ & 10 & $2(20)$ & 0 \\
$70-80$ & 2 & $0(0)$ & 0 \\
$80-90$ & 1 & $0(0)$ & 100 \\
\hline Total & 178 & $51(28.65)$ & 0.83 \\
\hline
\end{tabular}

to have a major impact on people living in urban and rural areas of developing countries with inestimable morbidity and mortality [14]. Epidemics have been reported in Sri Lanka in 2008 and in the Philippines in 2009 [9]. It has been reported to be rampant in southern, central, eastern, and western India where heavy monsoon, animal rearing, unplanned urbanization, and agrarian way of life predispose to the infection [15]. The geographical location, climactic condition, and rich fauna of Nepal seem to be suitable for survival of leptospires. Thus leptospirosis could be a significant public health problem in Nepal and this study is designed to depict the disease scenario in Nepal.

\section{Materials and Methods}

The study was designed as a descriptive cross-sectional study and was carried out from July 2011 to November 2011 at National Public Health Laboratory, Teku, Kathmandu.

2.1. Study Population. Patients referred to National Public Health Laboratory for a test on leptospirosis were included into the study. About $5 \mathrm{~mL}$ of blood from adults and $3 \mathrm{~mL}$ from children aged 5 years or younger were collected and serum separated for further serological testing. Any icteric sample or sample exhibiting haemolysis, lipaemia, or microbial growth was excluded from the study.

2.2. Data Collection. A standardized form was used, to collect information from patients suspected of leptospirosis. Information regarding demographic details (age, sex), clinical features (fever, onset day, chills, malaise, myalgia, arthralgia, retro orbital pain, headache, anorexia, nausea, vomiting, abdominal pain, bleeding jaundice, and meningitis), animal or water contact/exposure, and occupation was obtained. Any suspected patient who has been handling farm animals like cows and buffaloes or handling pet dogs or playing with them was considered to have an animal contact. Similarly any suspected patient who has been swimming/bathing, washing clothes, and fishing in the river or working in water logged field was considered to have water contact.

2.3. Serological Study. Serum samples were subjected to IgM capture ELISA (Panbio Leptospira IgM ELISA from Inverness
TABLE 2: Animal and water contact pattern in leptospirosis.

\begin{tabular}{lcccc}
\hline $\begin{array}{l}\text { Contact } \\
\text { type }\end{array}$ & $\begin{array}{c}\text { Number of } \\
\text { cases }\end{array}$ & $\begin{array}{c}\text { Number of } \\
\text { positives (\%) }\end{array}$ & $\begin{array}{c}\text { \% in total } \\
\text { positives }\end{array}$ & $p$ value \\
\hline $\begin{array}{l}\text { Animal } \\
\text { contact }\end{array}$ & 117 & $\begin{array}{c}42 \\
(35.90)\end{array}$ & 82.35 & 0.003 \\
$\begin{array}{l}\text { Water } \\
\text { contact }\end{array}$ & 80 & $\begin{array}{c}30 \\
(37.5)\end{array}$ & 58.82 & 0.018 \\
\hline
\end{tabular}

Medical Innovations, Australia) to detect anti-Leptospira IgM antibody present in the samples. Those giving positive result were defined as laboratory confirmed leptospirosis.

2.4. Statistical Analysis. Data were analysed using SPSS version 17.0. Values were expressed as mean \pm standard deviation. Chi-square analysis was carried out and value of significance used for all statistical tests was $p<0.05$.

\section{Results}

Out of 225 serum samples from patients suspected of leptospirosis, 178 were fit for inclusion into the study out of which 51 (28.65\%) were positive for anti-Leptospira IgM antibody. Among the positives, 33 (64.70\%) were males and $18(35.30 \%)$ were females but the difference was statistically insignificant ( $p$ value 0.986 ). Most of the patients were adults with a median age of 26.50. The youngest age showing positive result was 5 years and the oldest age was 60 years. Most of the positive cases clustered in the age groups 20$30(35.29 \%)$ and $30-40(21.57 \%)$ whereas the disease was less prevalent in either extremes of age (Table 1).

Patients having animal contact were 117 out of which 42 were positive for anti-Leptospira IgM and out of 80 patients traced to have water contact 30 were positive for antiLeptospira IgM (Table 2).

58 patients have both animal and water contact while 39 patients have neither animal nor water contact. Both modes of contact were found to be significantly related to leptospirosis.

Symptoms were vague and varied with the most common being fever $(100 \%)$, headache $(78.43 \%)$, myalgia $(68.63 \%)$, abdominal pain (31.37\%), vomiting (23.53\%), jaundice (17.65\%), and diarrhoea (15.69\%). Conjunctival suffusion, 
TABLE 3: Clinical features in leptospirosis.

\begin{tabular}{lcc}
\hline Clinical signs/symptoms & Number of cases among positives showing the symptoms & \% of total positive Cases \\
\hline Fever & 51 & 100 \\
Headache & 40 & 78.43 \\
Myalgia & 35 & 68.63 \\
Vomiting & 12 & 23.53 \\
Diarrhoea & 8 & 15.69 \\
Jaundice & 9 & 17.65 \\
Abdominal pain & 16 & 31.37 \\
\hline
\end{tabular}

TABLE 4: Occupation wise distribution of leptospirosis.

\begin{tabular}{lccc}
\hline Occupation & Number of cases (\% of total cases) & Number of positives (\%) & \% in total positive cases \\
\hline Farmer & $57(32.02)$ & $23(40.35)$ & 45.10 \\
Student & $57(32.02)$ & $15(26.31)$ & 29.41 \\
Housewife & $29(16.29)$ & $7(24.14)$ & 13.72 \\
Business & $15(8.43)$ & $2(13.33)$ & 3.92 \\
Service & $14(7.86)$ & $4(28.57)$ & 7.84 \\
Unemployed & $6(3.37)$ & $0(0)$ & 0 \\
\hline Total & 178 & $51(28.65)$ & 100 \\
\hline
\end{tabular}

muscle tenderness, retro orbital pain, rashes, and meningitis could not be reported (Table 3 ).

Among the suspected cases most were farmers (32.02\%) and students (32.02\%) followed by housewives (16.29\%) while among the positive cases $45.10 \%$ were farmers followed by students $(29.41 \%)$ and housewives (13.72\%). Six of the cases could not be ascribed to any occupation (Table 4 ).

\section{Discussion}

The study was conducted at National Public Health Laboratory, Teku, Kathmandu, on samples referred for a test on leptospirosis. Leptospirosis is not routinely diagnosed in Nepal and it is very likely that only the samples that could not be attributed to other febrile illnesses were sent for a test on leptospirosis. This fact combined along with small sample size is likely the cause of apparently high positivity of $28.65 \%$ compared to the finding that only $4.1 \%$ of febrile cases reported to Patan Hospital in 2001 were attributed to leptospirosis [16].

In our study males were more affected than females. This could be because of higher exposure of males to risk factors like animal rearing, working in fields, and others like swimming in the rivers. The preponderance of leptospirosis in males is in agreement with the finding of other workers $[15,17-19]$. The clustering of the disease in people in their $20 \mathrm{~s}$ and 30 s looks reasonable as people from these age groups are more involved in agriculture and animal rearing and hence are more likely to be exposed to risk factors associated with leptospirosis.

In our study both animal and water contact were significantly associated with leptospirosis. This could be in part due to small sample size. Rearing cattle and/or buffaloes are a common practice in Nepal. These animals are often chronically colonized with pathogenic leptospires with frequent transmission to humans $[2,14,20,21]$. The most common modes of water contact were working in the fields, swimming, bathing, and/or washing clothes in the rivers. Common source outbreaks have been reported following swimming in contaminated water [7]. Our study coincided with the rice growing season. The characteristics of water in the rice fields are appropriate for the survival of leptospires [7].

The symptoms complained were vague and varied with the most common being fever along with headache, myalgia, vomiting, jaundice, and diarrhoea. Other symptoms of interest like conjunctival suffusion, retro orbital pain, muscle tenderness, rashes, and meningitis could not be reported in our study which makes our analysis of clinical features insufficient to ascribe any symptom or combinations significantly to leptospirosis.

Most of those affected were farmers (45.10\%) followed by students (29.41\%) and housewives (13.72\%). Leptospirosis has traditionally been considered a disease of farmers [14]. The study period coincided with rice growing season and occupational activities that require prolonged contact with water or mud are associated with greater risk of infection $[17,19]$. Rearing cattle and/or buffaloes go hand in hand with agricultural practices as most of the manures needed for the crops are supplied by these animals, hence putting farmers and housewives in a greater risk. Students are likely to get exposed to risk factors while swimming and/or bathing in rivers or ponds and many also accompany their parents in fields for agricultural works. Housewives in Nepal are generally involved in animal rearing and agricultural works. Leptospirosis has been frequently found in students and housewives $[15,22]$.

The limitations of the study include the use of single serum sample for ELISA instead of paired sera and the short duration and small sample size. We could not perform MAT of ELISA positive samples which would have given 
knowledge of infecting serovars. However we believe that the result of this study depicts the general features of the disease in Nepal.

\section{Conclusion}

Life style heavily dominated by agronomical and animal rearing practices in Nepal is conducive to the transmission of leptospirosis. The disease seems to be a significant public health problem in Nepal but is underdiagnosed and underestimated. In resource poor countries like Nepal where laboratories performing MAT or maintaining cultures are rarely available, serological tests like ELISA can be helpful in early diagnosis of the disease.

\section{Competing Interests}

The authors declare that there is no conflict of interests regarding the publication of this paper.

\section{Acknowledgments}

The authors are extremely grateful to all the staffs and workers of National Public Health Laboratory, Teku, Kathmandu, for their technical and nontechnical assistance during the study period.

\section{References}

[1] F. Merien, G. Baranton, and P. Perolat, "Invasion of vero cells and induction of apoptosis in macrophages by pathogenic Leptospira interrogans are correlated with virulence," Infection and Immunity, vol. 65, no. 2, pp. 729-738, 1997.

[2] A. F. B. Victoriano, L. D. Smythe, N. Gloriani-Barzaga et al., "Leptospirosis in the Asia Pacific region," BMC Infectious Diseases, vol. 9, article 147, 2009.

[3] J. Croda, J. G. R. Ramos, J. Matsunaga et al., "Leptospira immunoglobulin-like proteins as a serodiagnostic marker for acute leptospirosis," Journal of Clinical Microbiology, vol. 45, no. 5, pp. 1528-1534, 2007.

[4] P. N. Levett, "Leptospirosis," Clinical Microbiology Reviews, vol. 14, no. 2, pp. 296-326, 2001.

[5] E. S. Shang, M. M. Exner, T. A. Summers et al., "The rare outer membrane protein, OmpL1, of pathogenic Leptospira species is a heat-modifiable porin," Infection and Immunity, vol. 63, no. 8 , pp. 3174-3181, 1995.

[6] H. Laurichesse, F. Gourdon, H. L. Smits et al., "Safety and immunogenicity of subcutaneous or intramuscular administration of a monovalent inactivated vaccine against Leptospira interrogans serogroup Icterohaemorrhagiae in healthy volunteers," Clinical Microbiology and Infection, vol. 13, no. 4, pp. 395403, 2007.

[7] W. Tangkanakul, P. Tharmaphornpil, B. D. Plikaytis et al., "Risk factors associated with leptospirosis in Northeastern Thailand, 1998," American Journal of Tropical Medicine and Hygiene, vol. 63, no. 3-4, pp. 204-208, 2000.

[8] T. R. Oliveira, M. T. Longhi, Z. M. De Morais et al., "Evaluation of leptospiral recombinant antigens MPL17 and MPL21 for serological diagnosis of leptospirosis by enzyme-linked immunosorbent assays," Clinical and Vaccine Immunology, vol. 15, no. 11, pp. 1715-1722, 2008.
[9] R. A. Hartskeerl, M. Collares-Pereira, and W. A. Ellis, "Emergence, control and re-emerging leptospirosis: dynamics of infection in the changing world," Clinical Microbiology and Infection, vol. 17, no. 4, pp. 494-501, 2011.

[10] World Health Organization, Human Leptospirosis: Guidance for Diagnosis Surveillance and Control, World Health Organization, Malta, 2003.

[11] V. Wuthiekanun, W. Chierakul, D. Limmathurotsakul et al., "Optimization of culture of Leptospira from humans with leptospirosis," Journal of Clinical Microbiology, vol. 45, no. 4, pp. 1363-1365, 2007.

[12] G. C. Gussenhoven, H. Mawgvd, M. G. A. Goris et al., "LEPTO dipstick, a dipstick assay for detection of Leptospira-specific immunoglobulin $\mathrm{M}$ antibodies in human sera," Journal of Clinical Microbiology, vol. 35, pp. 92-97, 1996.

[13] M. D. Bajani, D. A. Ashford, S. L. Bragg et al., "Evaluation of four commercially available rapid serologic tests for diagnosis of leptospirosis," Journal of Clinical Microbiology, vol. 41, no. 2, pp. 803-809, 2003.

[14] E. R. Cachay and J. M. Vinetz, "A global research agenda for leptospirosis," Journal of Postgraduate Medicine, vol. 51, no. 3, pp. 174-178, 2005.

[15] S. Sethi, N. Sharma, N. Kakkar et al., "Increasing trends of leptospirosis in Northern India: A Clinico-Epidemiological Study," PLoS Neglected Tropical Diseases, vol. 4, no. 1, article e579, 2010.

[16] D. R. Murdoch, C. W. Woods, M. D. Zimmerman et al., "The etiology of febrile illness in adults presenting to Patan Hospital in Kathmandu, Nepal," American Journal of Tropical Medicine and Hygiene, vol. 70, no. 6, pp. 670-675, 2004.

[17] C. B. L. Castellanos, R. G. Suarez, E. G. Figueroa, J. L. F. Allen, and J. E. De LaPena, "Risk factors and the prevalence of Leptospirosis infection in a rural community of Chipas, Mexico," Epidemiology \&Infection, vol. 131, no. 3, pp. 1149-1156, 2003.

[18] V. Chawla, T. H. Trivedi, and M. E. Yeolekar, "Epidemic of leptospirosis: an ICU experience," Journal of Association of Physicians of India, vol. 52, pp. 619-622, 2004.

[19] T. Panaphut, S. Domrongkitchaiporn, and B. Thinkamrop, "Prognostic factors of death in leptospirosis: a prospective cohort study in Khon Kaen, Thailand," International Journal of Infectious Diseases, vol. 6, no. 1, pp. 52-59, 2002.

[20] P. S. Cheema, S. K. Srivastava, R. Amutha, S. Singh, H. Singh, and M. Sandey, "Detection of pathogenic leptospires in animals by PCR based on lipL21 and lipL32 genes," Indian Journal of Experimental Biology, vol. 45, no. 6, pp. 568-573, 2007.

[21] C. D. Gamage, N. Koizumi, A. K. C. Perera et al., "Carrier status of leptospirosis among cattle in Sri Lanka: a zoonotic threat to public health," Transboundary and Emerging Diseases, vol. 61, no. 1, pp. 91-96, 2014.

[22] P. Vijayachari, A. P. Sugunan, M. V. Murhekar, S. Sharma, and S. C. Sehgal, "Leptospirosis among schoolchildren of the Andaman/Nicobar Islands, India: low levels of morbidity and mortality among pre-exposed children during an epidemic," Epidemiology and Infection, vol. 132, no. 6, pp. 1115-1120, 2004. 

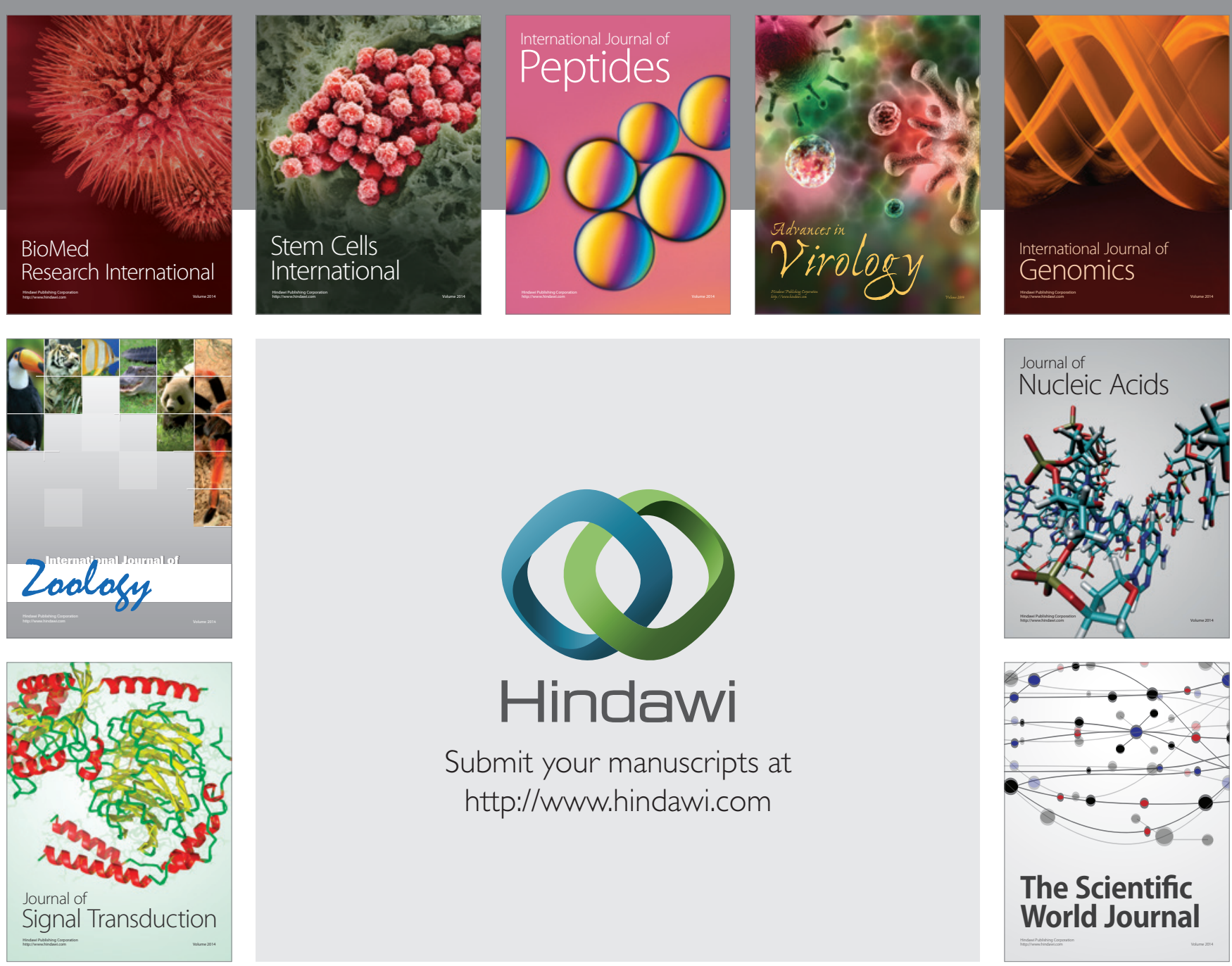

Submit your manuscripts at

http://www.hindawi.com
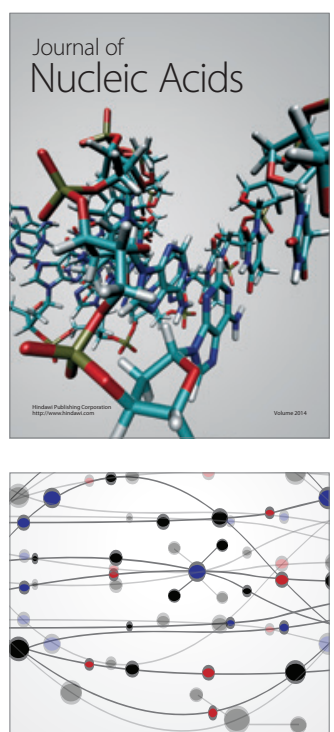

The Scientific World Journal
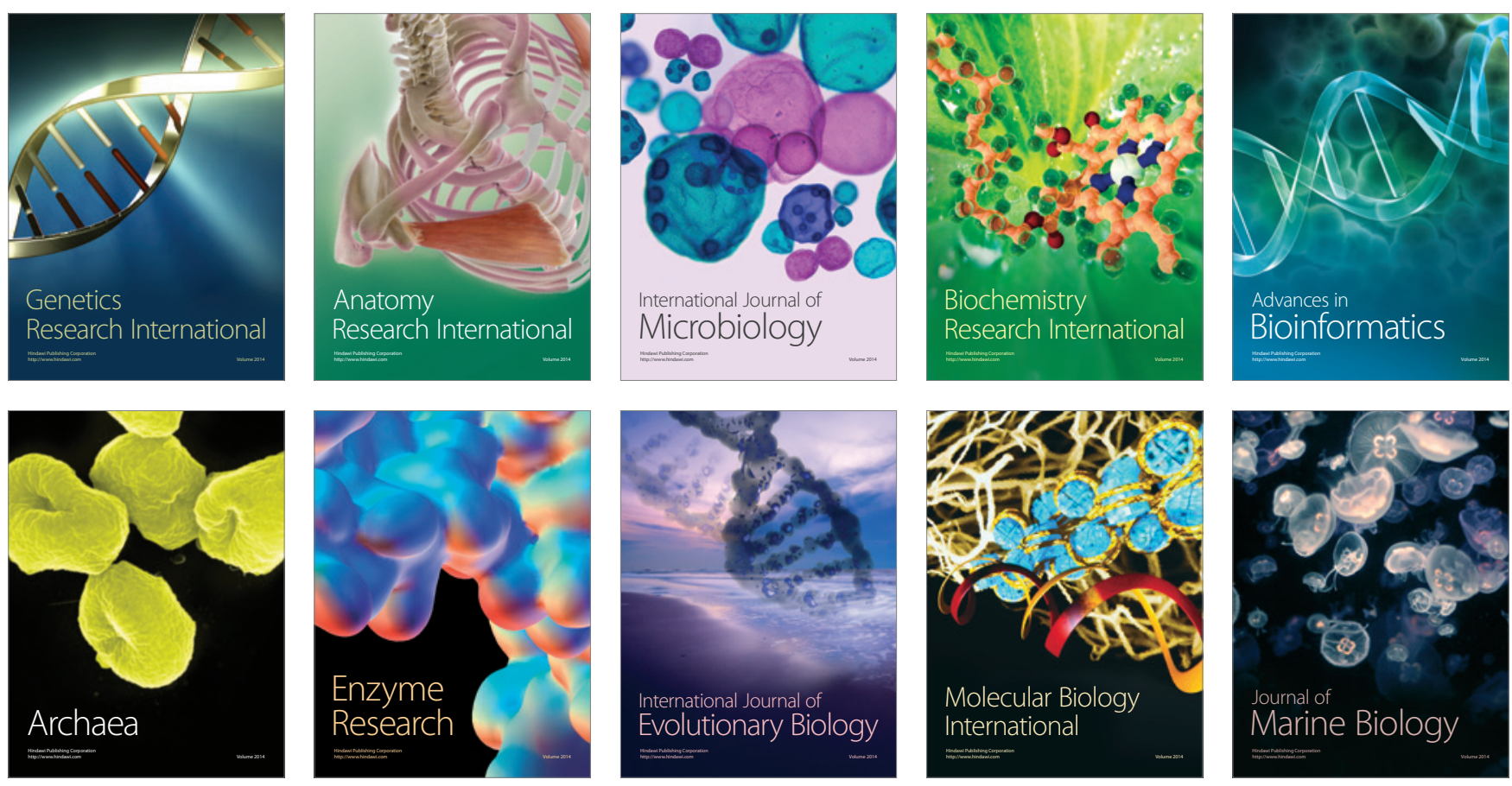